\title{
BACH1 mediates the antioxidant properties of aged garlic extract (Review)
}

\author{
TADAMITSU TSUNEYOSHI
}

Central Research Institute, Wakunaga Pharmaceutical Co. Ltd., Akitakata, Hiroshima 739-1195, Japan

Received July 19, 2019; Accepted August 21, 2019

DOI: $10.3892 /$ etm.2019.8380

\begin{abstract}
In clinical studies, aged garlic extract (AGE) has been shown to improve endothelial dysfunction. The activation of nuclear factor erythroid 2 like 2 (Nrf2)-dependent gene expression is a proposed mechanism for maintaining vascular homeostasis. $S$-1-propenylcysteine (S1PC) and $S$-allylcysteine (SAC) are two predominant sulfur-containing amino acids present in AGE. However, it remains unclear as to whether the two sulfur amino acids activate Nrf2 in cells. Nitric oxide (NO) is an important signaling molecule and one of the activators of the Nrf2 pathway. In a previous study, we examined the effects of the two sulfur amino acids on NO signaling for modulating the Nrf2-dependent antioxidant response. Neither S1PC nor SAC were found to affect the expression of Nrf2-regulated genes, such as heme oxygenase-1 (HMOX1) in human umbilical vein endothelial cells. However, S1PC was found to augment HMOX1 expression, induced by NO donors, such as NOR3. NOR3 was found to induce the nuclear accumulation of NRF2 protein and concomitantly enhance the degradation of BTB domain and $\mathrm{CNC}$ homolog 1 (BACH1), a transcriptional repressor that competes with NRF2. Notably, on our previous study, S1PC enhanced the NOR3-induced downregulation of BACH1, but did not further enhance the NOR3-induced accumulation of NRF2. The findings of that study indicated that the S1PC-induced degradation of BACH1 may provide a basis for the antioxidant effects of AGE. Thus, in this review, we aimed to provide a current overview of the antioxidant effects of AGE and sulfur-containing amino acids.
\end{abstract}

Correspondence to: Dr Tadamitsu Tsuneyoshi, Central Research Institute, Wakunaga Pharmaceutical Co. Ltd., 1624 Shimokotachi, Koda-cho, Akitakata, Hiroshima 739-1195, Japan

E-mail: tsuneyoshi_td@wakunaga.co.jp

Key words: S-1-propenylcysteine, BTB domain and CNC homolog 1, nuclear factor erythroid 2 like 2, heme oxygenase-1, nitric oxide, garlic, aged garlic extract

\section{Contents}

1. Antioxidant effects of aged garlic extract

2. Sulfur amino acids are known to increase resistance to oxidative stress by modulating the Nrf2/SKN-1 pathway

3. Known synergistic effect of S1PC and NO-donors on antioxidant gene expression

4. Evidence of the induction of BACH1 downregulation by S1PC in a NO-dependent manner

5. Bach1-deficient mice have been reported to exhibit a resistance phenotype in disease models

6. Conclusions and future perspectives

\section{Antioxidant effects of aged garlic extract}

The intake of raw garlic or its preparations has been shown to mitigate multiple risk factors associated with cardiovascular diseases (1). In clinical studies, aged garlic extract (AGE) has been shown to improve endothelial dysfunction that is considered as an early marker of atherosclerosis $(2,3)$. One possible mechanism underlying endothelial dysfunction is an increase in reactive oxygen species (ROS) generated by an enhanced energy metabolism or chronic inflammation. The antioxidant properties of AGE have been proposed to play a role in preventing endothelial dysfunction (4). AGE has been shown to contain organosulfur compounds, polyphenols and Maillard reaction products, such as $\mathrm{N} \alpha$-(1-deoxy-D-fructos-1-yl)-L-arginine. These chemicals have radical scavenging properties in vitro; however, the mechanisms through which these chemicals exert their antioxidant effects in vivo remain unclear $(5,6)$. The activation of nuclear factor erythroid 2 like 2 (Nrf2)-dependent gene expression has also been proposed as a mechanism for the maintenance of vascular homeostasis via the enhancement of the cellular defense mechanism against oxidative stresses (7). A recent study using canines demonstrated that the administration of AGE upregulated the gene expression levels of canine Nrf2 and phase II antioxidant enzymes (8).

2. Sulfur amino acids are known to increase resistance to oxidative stress by modulating the Nrf2/SKN-1 pathway

Garlic is rich in organosulfur compounds which are believed to be responsible for most of its pharmacological properties. $S$-1-propenylcysteine (S1PC) and $S$-allylcysteine (SAC) are 
Table I. Effects of Bachl deficiency in mouse disease models.

Disease models Results of Bachl (-/-) mice (Refs.)

Atherosclerosis, apolipoprotein E knockout mice fed a high-fat diet

Myocardial infarction, ischemia/reperfusion injury

Heart failure, pressure overload induced hypertrophy

Diabetes, alloxan-induced oxidative stress model

Inflammatory bowel diseases, trinitrobenzene sulfonic acid (TNBS)-induced colitis

Steatohepatitis, methionine-choline deficient diet model
Total atherosclerotic plaque area was reduced (14).

Myocardial infarction was reduced (16).

Left ventricular hypertrophy was inhibited (17).

Oxidative stress-induced apoptosis was reduced

in pancreatic $\beta$-cells (18).

TNBS-induced colitis was ameliorated (19).

Hepatic triglyceride and malondialdehyde was reduced (20).

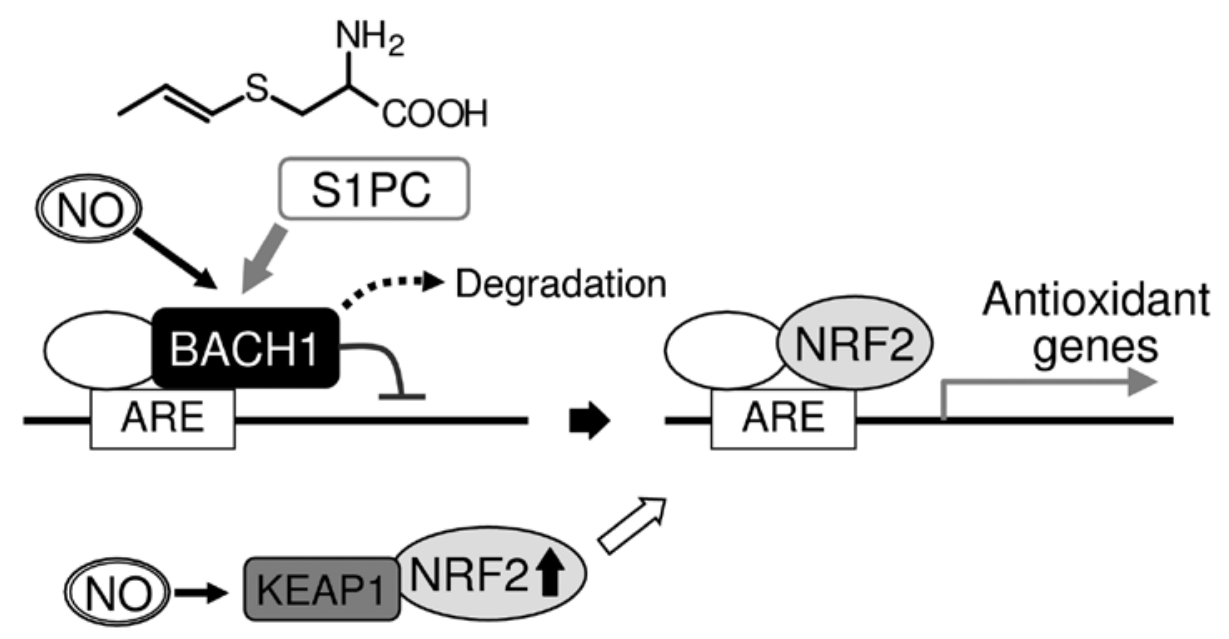

Figure 1. S-1-propenylcysteine augments BACH1 degradation and NRF2-regulated gene expression in a nitric oxide-dependent manner. BACH1, BTB domain and CNC homolog 1; NRF2, nuclear factor erythroid 2 like 2; KEAP1, Kelch-like ECH-associated protein 1; NO, nitric oxide; S1PC, $S$-1-propenylcysteine; ARE, antioxidant response element.

two sulfur amino acids predominantly found in AGE (5). In oxidative stress models using Caenorhabditis elegans, SAC has been shown to increase stress resistance and reduce the accumulation of ROS. These antioxidant effects have been shown to require the transcription factor, $\mathrm{SKN}-1$, that is the Nrf2 orthologue in mammals (9). Nrf2 is a transcription factor that regulates key antioxidant genes and phase II detoxification genes in mammals. Although the activation of Nrf2-dependent gene expression has been proposed as a mechanism for maintaining vascular homeostasis, it remains unclear as to whether SAC and S1PC activate Nrf2 in endothelial cells.

\section{Known synergistic effect of S1PC and NO-donors on an- tioxidant gene expression}

Nitric oxide (NO) is an important signaling molecule involved in maintaining vascular homeostasis and is also one of the activators of the Nrf2 pathway (10). Therefore, in a previous study, we examined the effects of SAC and S1PC on the signaling mechanism of NO in modulating the Nrf2-dependent antioxidant response in endothelial cells (11). Neither S1PC nor SAC, were found to independently affect the expression of Nrf2-regulated genes, such as heme oxygenase-1 (HMOX1) and glutamate-cysteine ligase modifier subunit $(G C L M)$ in human umbilical vein endothelial cells (HUVECs). However, S1PC was found to augment the expression of HMOXI and GCLM induced by NO donors, such as NOR3. In that study, SAC did not exert such synergistic effects with NO donors (11). In that previous study, we also confirmed the synergistic effect of S1PC with another NO donor, $S$-nitrosoglutathione (GSNO), on the expression of $H M O X 1$ in human aortic endothelial cells (HAECs) (11).

\section{Evidence of the induction of BACH1 downregulation by S1PC in a NO-dependent manner}

Under basal conditions, Nrf2 binds to Kelch-like ECH-associated protein 1 (Keap1) resulting in proteasomal degradation of Nrf2 in the cytoplasm. In the presence of oxidative stress, Nrf2 is released from the Keap1-dependent complex and accumulates in the nucleus. Nrf2 binds to antioxidant response elements (AREs) that are cis-elements essential for the expression of various antioxidant genes, including HMOXI and GCLM, whereas the BTB domain and CNC homolog 1 (BACH1) interacts with the AREs of the corresponding genes to prevent Nrf2 binding, thus inhibiting gene expression (12). In a previous study, in order to examine the synergistic effects of S1PC and NO in the context of antioxidant 
gene expression, we analyzed the protein expression of NRF2 and BACH1. A significant accumulation of NRF2 was not observed in HUVECs treated with S1PC alone. NOR3 was found to induce NRF2 accumulation; however, cells co-treated with NOR3 and S1PC did not exhibit any significant differences in NRF2 protein levels compared to cells treated only with NOR3 (11). NOR3 was also found to induce a reduction in BACH1 protein levels in HUVECs, whereas S1PC did not affect BACH1 levels. However, in that study, it was found that S1PC enhances BACH1 downregulation upon co-treatment with NOR3 (11). In that same study, to further evaluate the role of BACH1 in the synergistic effects of S1PC and NO-donors, we used HUVECs transfected with siRNA targeting $B A C H 1$. Compared to cells treated with NOR3 only, the suppression of $B A C H 1$ did not result in any significant enhancement in HMOX1 and GCLM expression upon co-treatment with S1PC and NOR3 (11). These results indicated that BACH1 plays a pivotal role in the synergistic effects of S1PC and NO donors on the expression of antioxidant genes.

\section{Bach1-deficient mice have been reported to exhibit a re- sistance phenotype in disease models}

Bach1-deficient mice are viable and fertile with no obvious phenotypic abnormalities under normal conditions, but compared to normal mice, these mice exhibit an enhanced expression of Hmoxl in diverse tissues (13). As the enhanced expression of Hmoxl is expected to exert antioxidant effects, Bachl-deficient mice have been studied in several disease models considered to involve oxidative stress. In apolipoprotein E-deficient mice that function as an atherosclerosis-prone model, Bachl-deficiency has been shown to reduce the plaque area and the excretion of 8 -iso-PG F2 $\alpha$, a marker of the systemic oxidative stress level (14). In several disease models listed in Table I, Bachl deficiency has been shown to exhibit a resistance phenotype. These results indicate that $\mathrm{BACH} 1$ downregulation might have potential therapeutic applications. However, promising lead compounds with a potential to reduce Bach1 levels have not yet been found, apart from heme-related compounds that also have the potential to produce ROS (15).

\section{Conclusions and future perspectives}

S1PC, a sulfur amino acid present in AGE, has the unique property of downregulating BACH1 in a NO-dependent manner and enhancing the expression of antioxidant genes reciprocally regulated by NRF2 and BACH1 (Fig. 1). Therefore, the interaction of S1PC and $\mathrm{BACH} 1$ could provide insight into the mechanisms through which AGE exerts its antioxidant effects in vivo.

\section{Acknowledgements}

The author would like to thank Dr Takami Oka and Dr Koichi Tamura (Wakunaga Pharmaceutical Co. Ltd.) for providing insight and valuable discussions pertaining to this review.

\section{Funding}

No funding was received.

\section{Availability of data and materials}

Not applicable.

\section{Authors' contributions}

TT designed the review and wrote the manuscript. The author has read and approved the final manuscript.

\section{Ethics approval and consent to participate}

Not applicable.

\section{Patient consent for publication}

Not applicable.

\section{Competing interests}

The author declares that they have no competing interests.

\section{References}

1. Banerjee SK and Maulik SK: Effect of garlic on cardiovascular disorders: A review. Nutr J 1: 4, 2002.

2. Budoff MJ, Ahmadi N, Gul KM, Liu ST, Flores FR, Tiano J, Takasu J, Miller E and Tsimikas S: Aged garlic extract supplemented with $\mathrm{B}$ vitamins, folic acid and L-arginine retards the progression of subclinical atherosclerosis: A randomized clinical trial. Prev Med 49: 101-107, 2009.

3. Weiss N, Ide N, Abahji T, Nill L, Keller C and Hoffmann U: Aged garlic extract improves homocysteine-induced endothelial dysfunction in macro- and microcirculation. J Nutr 136 (Suppl 3): 750S-754S, 2006.

4. Weiss N, Papatheodorou L, Morihara N, Hilge R and Ide N: Aged garlic extract restores nitric oxide bioavailability in cultured human endothelial cells even under conditions of homocysteine elevation. J Ethnopharmacol 145: 162-167, 2013.

5. Kodera Y, Ushijima M, Amano H, Suzuki JI and Matsutomo T: Chemical and biological properties of $S$-1-propenyl-1-cysteine in aged garlic extract. Molecules 22: 22, 2017.

6. Morihara N, Hayama M and Fujii H: Aged garlic extract scavenges superoxide radicals. Plant Foods Hum Nutr 66: 17-21, 2011.

7. Takabe W, Warabi E and Noguchi N: Anti-atherogenic effect of laminar shear stress via Nrf2 activation. Antioxid Redox Signal 15: 1415-1426, 2011.

8. Yamato $\mathrm{O}$, Tsuneyoshi T, Ushijima $\mathrm{M}$, Jikihara $\mathrm{H}$ and Yabuki A: Safety and efficacy of aged garlic extract in dogs: Upregulation of the nuclear factor erythroid 2-related factor 2 (Nrf2) signaling pathway and Nrf2-regulated phase II antioxidant enzymes. BMC Vet Res 14: 373, 2018.

9. Ogawa T, Kodera Y, Hirata D, Blackwell TK and Mizunuma M: Natural thioallyl compounds increase oxidative stress resistance and lifespan in Caenorhabditis elegans by modulating SKN-1/Nrf. Sci Rep 6: 21611, 2016.

10. Liu XM, Peyton KJ, Ensenat D, Wang H, Hannink M, Alam J and Durante W: Nitric oxide stimulates heme oxygenase-1 gene transcription via the Nrf2/ARE complex to promote vascular smooth muscle cell survival. Cardiovasc Res 75: 381-389, 2007.

11. Tsuneyoshi T, Kunimura K and Morihara N: S-1-Propenylcysteine augments $\mathrm{BACH} 1$ degradation and heme oxygenase 1 expression in a nitric oxide-dependent manner in endothelial cells. Nitric Oxide 84: 22-29, 2019.

12. Reichard JF, Motz GT and Puga A: Heme oxygenase-1 induction by NRF2 requires inactivation of the transcriptional repressor BACH1. Nucleic Acids Res 35: 7074-7086, 2007.

13. Sun J, Hoshino H, Takaku K, Nakajima O, Muto A, Suzuki H, Tashiro S, Takahashi S, Shibahara S, Alam J, et al: Hemoprotein Bach1 regulates enhancer availability of heme oxygenase-1 gene. EMBO J 21: 5216-5224, 2002. 
14. Watari Y, Yamamoto Y, Brydun A, Ishida T, Mito S, Yoshizumi M, Igarashi K, Chayama K, Ohshima T and Ozono R: Ablation of the bach1 gene leads to the suppression of atherosclerosis in bach1 and apolipoprotein E double knockout mice. Hypertens Res 31: 783-792, 2008.

15. Igarashi $\mathrm{K}$ and Watanabe-Matsui $\mathrm{M}$ : Wearing red for signaling: The heme-bach axis in heme metabolism, oxidative stress response and iron immunology. Tohoku J Exp Med 232: 229-253, 2014.

16. Yano Y, Ozono R, Oishi Y, Kambe M, Yoshizumi M, Ishida T, Omura S, Oshima T and Igarashi K: Genetic ablation of the transcription repressor Bach1 leads to myocardial protection against ischemia/reperfusion in mice. Genes Cells 11: 791-803, 2006.

17. Mito S, Ozono R, Oshima T, Yano Y, Watari Y, Yamamoto Y, Brydun A, Igarashi K and Yoshizumi M: Myocardial protection against pressure overload in mice lacking Bach1, a transcriptional repressor of heme oxygenase-1. Hypertension 51: 1570-1577, 2008.
18. Kondo K, Ishigaki Y, Gao J, Yamada T, Imai J, Sawada S, Muto A, Oka Y, Igarashi K and Katagiri H: Bach1 deficiency protects pancreatic $\beta$-cells from oxidative stress injury. Am J Physiol Endocrinol Metab 305: E641-E648, 2013.

19. Harusato A, Naito Y, Takagi T, Uchiyama K, Mizushima K, Hirai Y, Higashimura Y, Katada K, Handa O, Ishikawa T, et al: BTB and CNC homolog 1 (Bach1) deficiency ameliorates TNBS colitis in mice: Role of $\mathrm{M} 2$ macrophages and heme oxygenase-1. Inflamm Bowel Dis 19: 740-753, 2013.

20. Inoue M, Tazuma S, Kanno K, Hyogo H, Igarashi K and Chayama K: Bach1 gene ablation reduces steatohepatitis in mouse MCD diet model. J Clin Biochem Nutr 48: 161-166, 2011.

This work is licensed under a Creative Commons Attribution-NonCommercial-NoDerivatives 4.0 International (CC BY-NC-ND 4.0) License. 